Acta Crystallographica Section D

\section{Biological Crystallography}

ISSN 1399-0047

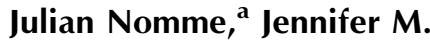
Murphy, ${ }^{b, c} \neq$ Ying $\mathrm{Su}^{a}$

Natasha D. Sansone, ${ }^{a} \S$

Amanda L. Armijo,, ${ }^{b, c}$ Steven T. Olson, ${ }^{d}$ Caius Radu ${ }^{\text {b,c }}$ and Arnon Lavie $^{\mathrm{a} *}$

${ }^{a}$ Department of Biochemistry and Molecular Genetics, University of Illinois at Chicago, Chicago, IL 60607, USA, 'b Department of Molecular and Medical Pharmacology, University of California, Los Angeles, CA 90095 , USA, cAhmanson Translational Imaging Division, University of California, Los Angeles, CA 90095, USA, and ${ }^{\mathbf{d}}$ Center for Molecular Biology of Oral Diseases, University of Illinois at Chicago, Chicago, IL 60612, USA

₹ Present address: UCLA, Crump Institute for Molecular Imaging CNSI, 570 Westwood Plaza, Building 114 Box 951770, Mail Code 177010, Los Angeles, CA 90095-1770, USA.

$\S$ Present address: Loyola University Medical Center, SSOM Building 112/Room \#219, 2160 South 1st Avenue, Maywood, IL 60153, USA.

Correspondence e-mail: lavie@uic.edu

\title{
Structural characterization of new deoxycytidine kinase inhibitors rationalizes the affinity- determining moieties of the molecules
}

Deoxycytidine kinase (dCK) is a key enzyme in the nucleoside salvage pathway that is also required for the activation of several anticancer and antiviral nucleoside analog prodrugs. Additionally, dCK has been implicated in immune disorders and has been found to be overexpressed in several cancers. To allow the probing and modulation of $\mathrm{dCK}$ activity, a new class of small-molecule inhibitors of the enzyme were developed. Here, the structural characterization of four of these inhibitors in complex with human dCK is presented. The structures reveal that the compounds occupy the nucleoside-binding site and bind to the open form of dCK. Surprisingly, a slight variation in the nature of the substituent at the 5-position of the thiazole ring governs whether the active site of the enzyme is occupied by one or two inhibitor molecules. Moreover, this substituent plays a critical role in determining the affinity, improving it from $>700$ to $1.5 \mathrm{n} M$ in the best binder. These structures lay the groundwork for future modifications that would result in even tighter binding and the correct placement of moieties that confer favorable pharmacodynamics and pharmacokinetic properties.

\section{Introduction}

Biosynthesis of deoxyribonucleotides, which are required for DNA replication and repair, can be achieved through either de novo synthesis or salvage pathways (Reichard, 1988). The de novo synthesis pathway uses glucose and amino acids to first produce ribonucleotides (Evans \& Guy, 2004) that are then processed to deoxyribonucleotides by ribonucleotide reductase (Fairman et al., 2011). Alternatively, the nucleosidesalvage pathway recycles bases and nucleosides originating from the degradation of RNA and DNA. The salvaged bases and nucleosides can then be converted back into nucleotides for DNA synthesis (Arnér \& Eriksson, 1995). Owing to crosstalk and compensatory potential of the enzymes participating in these synthetic pathways, the functional in vivo role of each individual enzyme is ambiguous.

A key member of the salvage pathway is deoxycytidine kinase (dCK), a dimeric enzyme composed of 260 residues per protomer. This cytosolic nucleoside kinase catalyzes the 5 -phosphorylation of deoxycytidine (dC), deoxyadenosine (dA) and deoxyguanosine (dG), with either ATP or UTP serving as the phosphoryl donor (Sabini, Hazra, Ort et al., 2008). Additionally, dCK phosphorylates, and as a result activates, numerous anticancer and antiviral nucleoside analogues such as fludarabine (Van den Neste et al., 2005), clofarabine (Montgomery et al., 1992), gemcitabine (Ruiz van
Received 30 May 2013

Accepted 9 September 2013

PDB References: deoxycytidine kinase, complex with F1, $4 \mathrm{jlj}$; complex with F2, 4jlk;

complex with F3, 4jlm;

complex with F4, 4jln 
Haperen et al., 1994) and lamivudine (Kewn et al., 2000). Although the enzymatic function of dCK in DNA-precursor biosynthesis and nucleoside-analog activation has been well established, its physiological implications remain unclear.

The biological function of proteins is often explored using approaches such as small interfering RNA (siRNA), gene deletion or the use of small-molecule inhibitors directed against the protein of interest. To investigate the possible role of dCK in lymphopoiesis, Toy and collaborators generated dCK knockout mice (Toy et al., 2010). Using this powerful tool, they could demonstrate that normal lymphocyte development requires dCK activity (Austin et al., 2012). However, the genedeletion approach does not permit the evaluation of the temporal function of the gene product. In contrast, smallmolecule inhibitors offer an advantage by providing control over when the activity of the enzyme under study is eliminated. Prior to our work, the sole report of potent dCK inhibitors originated from Lexicon Pharmaceuticals, whose goal was to better understand the role of dCK in immunerelated diseases (Jessop et al., 2009; Tarver et al., 2009; Yu et al., 2010).

In addition to probing the in vivo function of dCK, inhibitors of this enzyme could play a role in immunodeficiency syndromes owing to adenosine deaminase or purine phosphorylase deficiencies as well as in cancer treatment (Joachims et al., 2008; Weng et al., 2013). Notably, overexpression of dCK has been observed in several cancers (Eriksson et al., 1994). While dCK activity is not required for cell proliferation (the use of dCK inhibitors would not prevent cancer growth), inhibition of dCK in cancer cells would generate an imbalance in nucleotide-precursor synthesis. This could potentially induce DNA-synthesis/repair defects and ultimately cell death when combined with inhibitors of the de novo pathway and/or DNA-damaging agents.

Owing to the aforementioned critical roles played by dCK, we initiated a program to identify small-molecule dCK inhibitors. A report (Murphy et al., 2013) details a high-throughput screen (HTS) that resulted in two hit compounds; a subsequent structure-activity relationship (SAR) analysis was performed which yielded potent $\mathrm{dCK}$ inhibitors with in vivo efficacy. Here, we present the crystal structures of the human dCK enzyme in complex with the best of what we refer to as the F-series of dCK inhibitors. The X-ray structures reveal how the compounds interact with $\mathrm{dCK}$ and rationalize the different binding affinities of the inhibitors. Moreover, the structures are being exploited to guide future modifications to the F-series inhibitors for attaining desired in vivo properties such as metabolic stability and biodistribution/absorption without perturbing the already attained nanomolar affinity.

\section{Materials and methods}

\subsection{Materials}

General laboratory reagents were purchased from Fisher (Pittsburgh, Pennsylvania, USA) and Sigma-Aldrich (St Louis, Missouri, USA). All nucleosides and nucleotides were obtained from Sigma. All inhibitors were synthesized at UCLA (Murphy et al., 2013).

\subsection{Protein expression and purification}

The human dCK variant used in these studies contained five mutations: four solvent-exposed cysteine residues are mutated to serines $(\mathrm{C} 9 \mathrm{~S} / \mathrm{C} 45 \mathrm{~S} / \mathrm{C} 59 \mathrm{~S} / \mathrm{C} 146 \mathrm{~S}$; referred to as $\mathrm{C} 4 \mathrm{~S})$ and Ser74 is mutated to a glutamic acid. Our previous work showed that the C4S-dCK variant generates better quality crystals without altering the three-dimensional structure of dCK or its enzymatic activity (Sabini et al., 2007). The S74E mutation serves to mimic the phosphorylated state of this serine residue. When we refer to $\mathrm{dCK}$ in this report, we mean the C4S-S74E-dCK variant. dCK was expressed in Escherichia coli BL21 C41(DE3) cells using a pET-14b vector; the cells were grown in $2 \times$ YT medium and induced with $0.1 \mathrm{~m} M$ IPTG for $4 \mathrm{~h}$ at $310 \mathrm{~K}$. The cells were harvested and the pellet was lysed by sonication. The lysate was cleared by centrifugation at $30000 \mathrm{rev} \min ^{-1}$ for $1 \mathrm{~h}$ at $277 \mathrm{~K}$ and the supernatant was loaded onto a $5 \mathrm{ml}$ HisTrap nickel-affinity column (GE Healthcare). The column was washed with $300 \mathrm{ml}$ of a buffer composed of $25 \mathrm{~m} M$ Tris- $\mathrm{HCl} \mathrm{pH} \mathrm{7.5,500} \mathrm{m} M \mathrm{NaCl}, 30 \mathrm{~m} M$ imidazole. The bound protein was eluted with the same buffer but containing $250 \mathrm{mM}$ imidazole and was further purified by gel filtration using an S-200 column in a buffer consisting of $25 \mathrm{~m} M$ HEPES $\mathrm{pH}$ 7.5, $200 \mathrm{~m} M$ sodium citrate, $2 \mathrm{~m} M$ EDTA, $3 \mathrm{~m} M$ DTT. The protein fractions were pooled, concentrated, aliquoted, flash-frozen in liquid nitrogen and stored at $193 \mathrm{~K}$ until use.

\subsection{Kinetic assay}

The phosphorylation activity of dCK was determined using a spectroscopic NADH-dependent enzyme-coupled assay (Agarwal et al., 1978; Sabini et al., 2003). All measurements were taken in triplicate at $310 \mathrm{~K}$ in a buffer consisting of $100 \mathrm{~m} M$ Tris pH 7.5, $200 \mathrm{~m} M \mathrm{KCl}, 5 \mathrm{~m} M \mathrm{MgCl}_{2}, 0.5 \mathrm{~m} M$ EDTA, $0.8 \mathrm{~m} M$ phosphoenolpyruvate, $0.4 \mathrm{~m} M$ NADH with $50 \mathrm{n} M$ dCK and $1 \mathrm{~m} M$ ATP. To determine the $\mathrm{IC}_{50}^{\text {app }}$ values, we measured the observed rate $\left(V_{\text {obs }}\right)$ values in the absence and the presence of the different inhibitors at a fixed saturated nucleoside concentration $(100 \mu M \mathrm{dC})$. The relative enzyme activity was plotted and $\mathrm{IC}_{50}^{\mathrm{app}}$ was determined using the equation

$$
V_{\text {obs }}=100 \times \mathrm{IC}_{50}^{\mathrm{app}} /\left(\mathrm{IC}_{50}^{\mathrm{app}}+[\mathrm{I}]_{\mathrm{o}}\right),
$$

where $\mathrm{IC}_{50}^{\mathrm{app}}$ is the concentration of the inhibitor necessary to inhibit $50 \%$ of dCK activity and $[\mathrm{I}]_{\mathrm{o}}$ is the inhibitor concentration. Owing to the relatively slow rate of $\mathrm{dC}$ phosphorylation by $\mathrm{dCK}$ (even at a saturating $\mathrm{dC}$ concentration), we were prevented from using a dCK concentration of below $50 \mathrm{n} M$.

The steady-state kinetic data were also fitted to an explicit inhibition model (see text). The equation for this model is a quadratic

$$
\begin{aligned}
V_{\text {obs }} & =V_{\mathrm{o}} \times\left(n \times[\mathrm{E}]_{\mathrm{o}}-[\mathrm{I}]_{\mathrm{o}}-K_{\mathrm{i}}^{\mathrm{app}}+\left\{\left([\mathrm{I}]_{\mathrm{o}}+K_{\mathrm{i}}^{\mathrm{app}}-n \times[\mathrm{E}]_{\mathrm{o}}\right)^{2}\right.\right. \\
& \left.\left.+4 \times K_{\mathrm{i}}^{\mathrm{app}} \times n \times[\mathrm{E}]_{\mathrm{o}}\right\}^{1 / 2}\right) /\left(2 \times n \times[\mathrm{E}]_{\mathrm{o}}\right),
\end{aligned}
$$


Table 1

Data-collection and refinement statistics.

Values in parentheses are for the last shell.

\begin{tabular}{|c|c|c|c|c|}
\hline Complex & F1 & F2 & F3 & F4 \\
\hline PDB code & $4 \mathrm{jlj}$ & $4 \mathrm{jlk}$ & $4 \mathrm{jlm}$ & $4 j \ln$ \\
\hline \multicolumn{5}{|l|}{ Data-collection statistics } \\
\hline $\mathrm{X}$-ray source & LS-CAT 21-ID-G & SER-CAT 22-BM & LS-CAT 21-ID-G & LS-CAT 21-ID-G \\
\hline Detector & MAR CCD 300 & MAR CCD 225 & MAR CCD 300 & MAR CCD 300 \\
\hline Wavelength $(\AA)$ & 0.97856 & 1.00000 & 0.97856 & 0.97856 \\
\hline Temperature $(\mathrm{K})$ & 100 & 100 & 100 & 100 \\
\hline Resolution $(\AA)$ & $2.00(2.12-2.00)$ & $1.89(2.01-1.89)$ & $2.18(2.31-2.18)$ & $2.15(2.25-2.15)$ \\
\hline \multicolumn{5}{|l|}{ No. of reflections } \\
\hline Observed & 219225 & 239787 & 157048 & 166951 \\
\hline Unique & 38798 & 44054 & 28925 & 29888 \\
\hline Completeness (\%) & $99.1(98.3)$ & $99.3(96.2)$ & $98.7(93.5)$ & $98.9(96.8)$ \\
\hline$R_{\text {merge }}^{\dagger}$ & $0.06(0.66)$ & $0.04(0.69)$ & $0.07(0.63)$ & $0.05(0.52)$ \\
\hline$\langle I / \sigma(I)\rangle$ & $14.0(2.3)$ & $22.4(2.5)$ & $13.6(2.3)$ & $18.2(2.8)$ \\
\hline Space group & $P 4_{1}$ & $P 4_{1}$ & $P 4_{1}$ & $P 4_{1}$ \\
\hline \multicolumn{5}{|l|}{ Unit-cell parameters $(\AA)$} \\
\hline$a=b$ & 68.99 & 68.59 & 68.51 & 68.65 \\
\hline$c$ & 123.18 & 120.71 & 121.28 & 120.05 \\
\hline Wilson $B$ factor $\left(\AA^{2}\right)$ & 49.5 & 39.1 & 55.1 & 50.5 \\
\hline \multicolumn{5}{|l|}{ Refinement statistics } \\
\hline Refinement program & REFMAC5 & REFMAC5 & REFMAC5 & REFMAC5 \\
\hline Twinning fraction & 0.5 & 0.5 & 0.5 & 0.5 \\
\hline$R_{\text {cryst }}$ & 0.17 & 0.17 & 0.17 & 0.20 \\
\hline$R_{\text {free }}$ & 0.21 & 0.21 & 0.24 & 0.25 \\
\hline Resolution range $(\AA)$ & $30-2.00$ & $30-1.89$ & $30-2.18$ & $30-2.15$ \\
\hline $\begin{array}{l}\text { Protein molecules per } \\
\text { asymmetric unit }\end{array}$ & 2 & 2 & 2 & 2 \\
\hline \multicolumn{5}{|l|}{ No. of atoms } \\
\hline \multicolumn{5}{|l|}{ Protein } \\
\hline Protomer $A$ & 1933 & 1927 & 1942 & 1928 \\
\hline Protomer $B$ & 1956 & 1967 & 1956 & 1970 \\
\hline Inhibitor & $27 \times 4$ & $28 \times 4$ & $29 \times 3$ & $30 \times 2$ \\
\hline UDP & $25 \times 2$ & $25 \times 2$ & $25 \times 2$ & $25 \times 2$ \\
\hline Water & 119 & 208 & 115 & 110 \\
\hline \multicolumn{5}{|l|}{ R.m.s. deviation from ideal $\neq$} \\
\hline Bond length $(\AA)$ & 0.012 & 0.012 & 0.013 & 0.008 \\
\hline Bond angles $\left({ }^{\circ}\right)$ & 1.602 & 1.602 & 1.741 & 1.336 \\
\hline \multicolumn{5}{|l|}{ Average $B$ factors $\left(\AA^{2}\right)$} \\
\hline \multicolumn{5}{|l|}{ Protein } \\
\hline Protomer $A$ & 44.1 & 34.3 & 51.5 & 45.8 \\
\hline Protomer $B$ & 45.1 & 34.4 & 52.5 & 46.7 \\
\hline \multicolumn{5}{|l|}{ Inhibitor } \\
\hline Protomer $A(301,302)$ & $48.0,51.0$ & $38.5,45.1$ & $50.4,36.4$ & $43.7,-$ \\
\hline Protomer $B(301,302)$ & $48.4,54.7$ & $36.2,44.2$ & $52.9,-$ & $38.4,-$ \\
\hline \multicolumn{5}{|l|}{ UDP } \\
\hline Protomer $A$ & 41.8 & 38.6 & 58.1 & 50.7 \\
\hline Protomer $B$ & 44.5 & 44.8 & 58.6 & 42.2 \\
\hline Waters & 40.3 & 34.8 & 43.8 & 40.2 \\
\hline \multicolumn{5}{|c|}{ Ramachandran plot\$, residues in $(\%)$} \\
\hline Most favored regions & 91.1 & 91.0 & 89.2 & 90.8 \\
\hline Additionally allowed regions & 8.0 & 8.3 & 10.1 & 8.7 \\
\hline Generously allowed regions & 0.7 & 0.7 & 0.7 & 0.2 \\
\hline Disallowed regions & 0.2 & 0.0 & 0.0 & 0.2 \\
\hline MolProbity score & 2.48 & 2.35 & 2.80 & 2.01 \\
\hline
\end{tabular}

$\dagger R_{\text {merge }}=\sum_{h k l} \sum_{i}\left|I_{i}(h k l)-\langle I(h k l)\rangle\right| / \sum_{h k l} \sum_{i} I_{i}(h k l)$. $\ddagger$ Engh \& Huber (1991). § Ramachandran statistics were defined using PROCHECK (Laskowski et al., 1993). ॠ As reported by MolProbity (http://molprobity.biochem.duke.edu/; Chen et al., 2010).

where $V_{\text {obs }}$ is the observed rate, $V_{\mathrm{o}}$ is the velocity in the absence of inhibitor, $n$ is a stoichiometric factor, $[\mathrm{E}]_{\mathrm{o}}$ is the dCK concentration, $[\mathrm{I}]_{\mathrm{o}}$ is the inhibitor concentration and $K_{\mathrm{i}}^{\text {app }}$ is the apparent inhibition constant.

Because compounds F3 and F4 bind with the highest affinity, the stoichiometric factor $n$ is easily determined by fitting the data to the quadratic equation. F3 and F4 give similar values of 0.77 and 0.82 , respectively. This means that when $\sim 0.8$ equivalents of the inhibitor are added, $100 \%$ of the enzyme has been inhibited. This suggests that the enzyme and inhibitor concentrations are each associated with an error and $n$ corrects the concentration of the enzyme to give 1:1 inhibition. The error can be in either the enzyme (not completely active) or the inhibitor or both. The similar correction factors for the two inhibitors suggests that there is a consistent error in the enzyme concentration of $\sim 20 \%$. This is not unreasonable.

Because F2 and F1 do not bind as tightly as F3 and F4, we fixed the stoichiometric factor at the average value of 0.8 to fit these data. This results in excellent fits for these inhibitors. As the activity measured in the absence of inhibitor is also associated with an error, we also fitted this value as a parameter. All the data for each titration were then normalized to the fitted value of activity in the absence of inhibitor. In addition, we weighted the data by the relative standard deviations of each point, although this did not make a large difference to the fits. All data were fitted using the KaleidaGraph software.

\subsection{Crystallization, $X$-ray data collection and refinement}

Crystals of human dCK in complex with inhibitors and UDP were grown at $285 \mathrm{~K}$ using the hanging-drop vapor-diffusion method. The conditions for all dCK-inhibitor complexes were as follows: $1 \mu \mathrm{l} \mathrm{dCK}$ protein at $10-17 \mathrm{mg} \mathrm{ml}^{-1}$ in complex with a 2.5-fold molar excess of inhibitor, $2 \mathrm{~m} M$ UDP and $5 \mathrm{mM} \mathrm{MgCl} 2$ was mixed with $1 \mu \mathrm{l}$ reservoir buffer solution. The reservoir solution consisted of $0.9-1.5 \mathrm{M}$ trisodium citrate dehydrate and $25 \mathrm{~m} M$ HEPES pH 7.5. Prior to data collection, crystals were soaked in mineral oil for cryoprotection. Diffraction data for dCK in complex with F2 were collected on SER-CAT beamline 22-BM at the Advanced Photon Source, Argonne National Laboratory. Data for all other complexes were collected on the Life Sciences Collaborative Access Team (LS- 
CAT) beamline 21-ID-G. Data were processed and scaled with $X D S$ and XSCALE (Kabsch, 2010). Structures were determined by molecular replacement with MOLREP (Vagin \& Teplyakov, 2010) using the dCK structure (PDB entry 3qen; Hazra et al., 2011) as a search model. Refinement was conducted using REFMAC (Murshudov et al., 2011) and model building using Coot (Emsley et al., 2010). All inhibitor coordinates and library descriptions were generated using the PRODRG server (Schüttelkopf \& van Aalten, 2004). All data sets were perfectly twinned and iterative refinements were

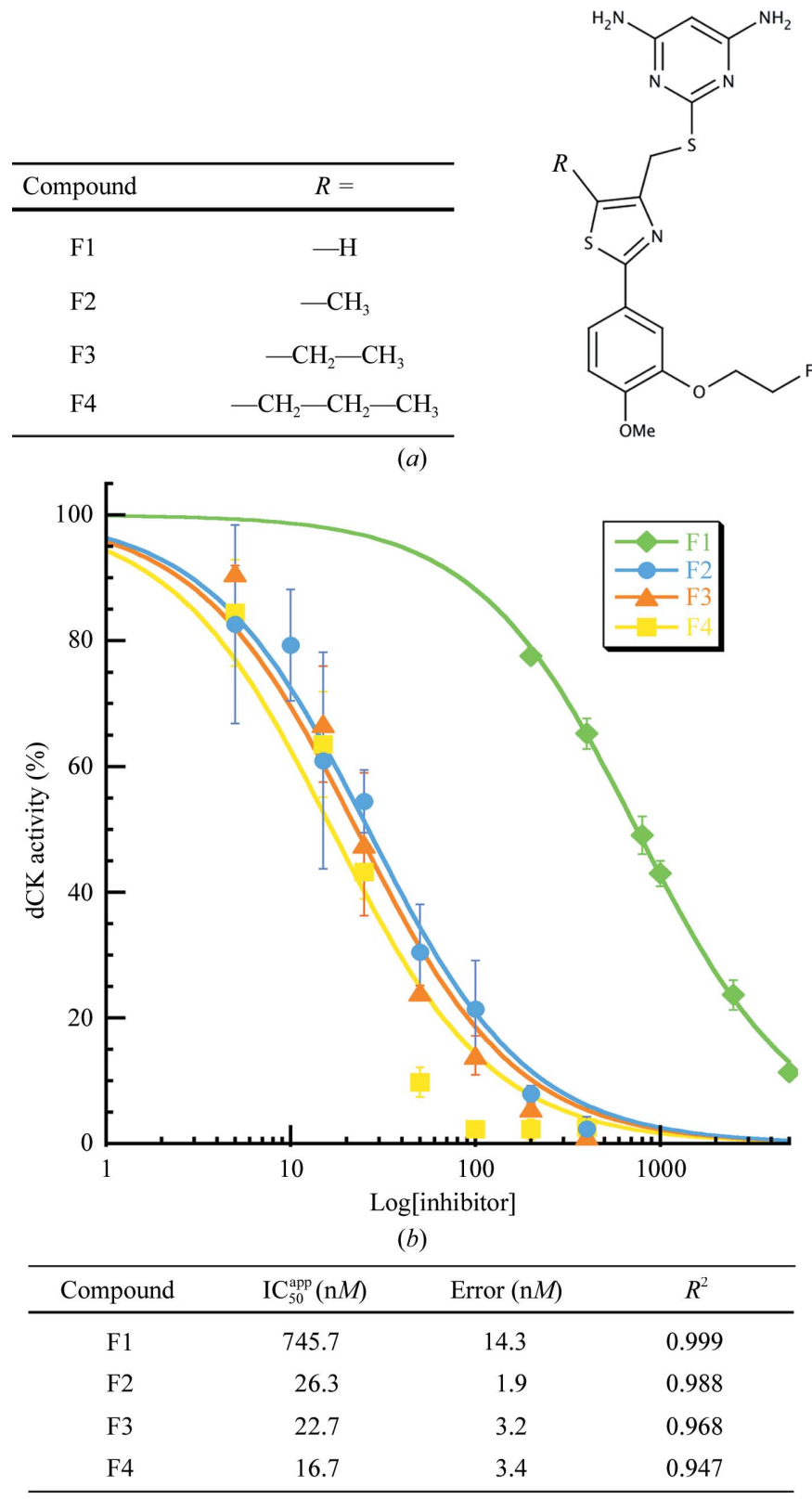

(c)

Figure 1

Schematic of the dCK inhibitors and their apparent $\mathrm{IC}_{50}$ values. (a) Schematic diagram of the F-series family of compounds and the nature of the substituent at the 5-position of the thiazole ring. (b) Data and curve fit used to determine the $\mathrm{IC}_{50}^{\text {app }}$ values. (c) $\mathrm{IC}_{50}^{\mathrm{app}}$ values, errors and coefficients of determination $\left(R^{2}\right)$. carried out using REFMAC with the Twin option active. Datacollection and refinement statistics are listed in Table 1. Structural figures were prepared using the PyMOL Molecular Graphics System (v.1.4.1; Schrödinger).

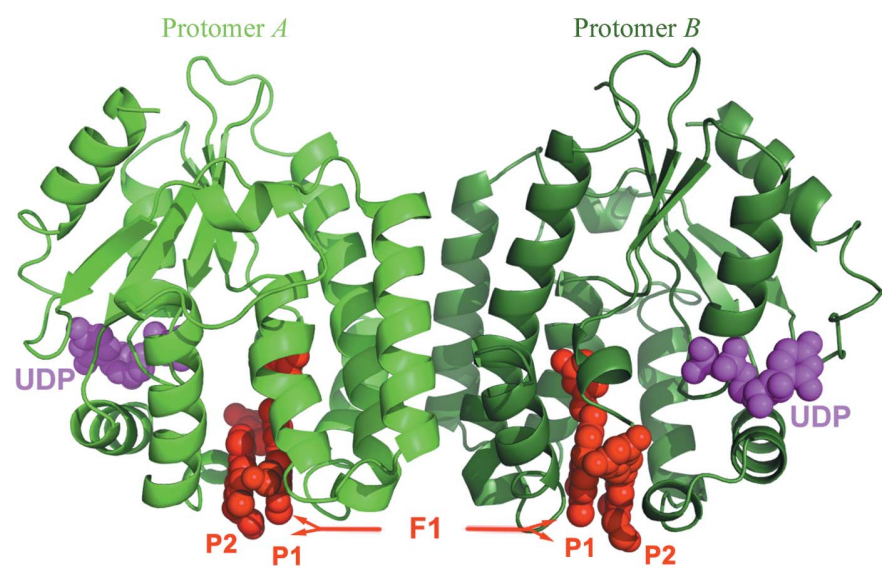

(a)

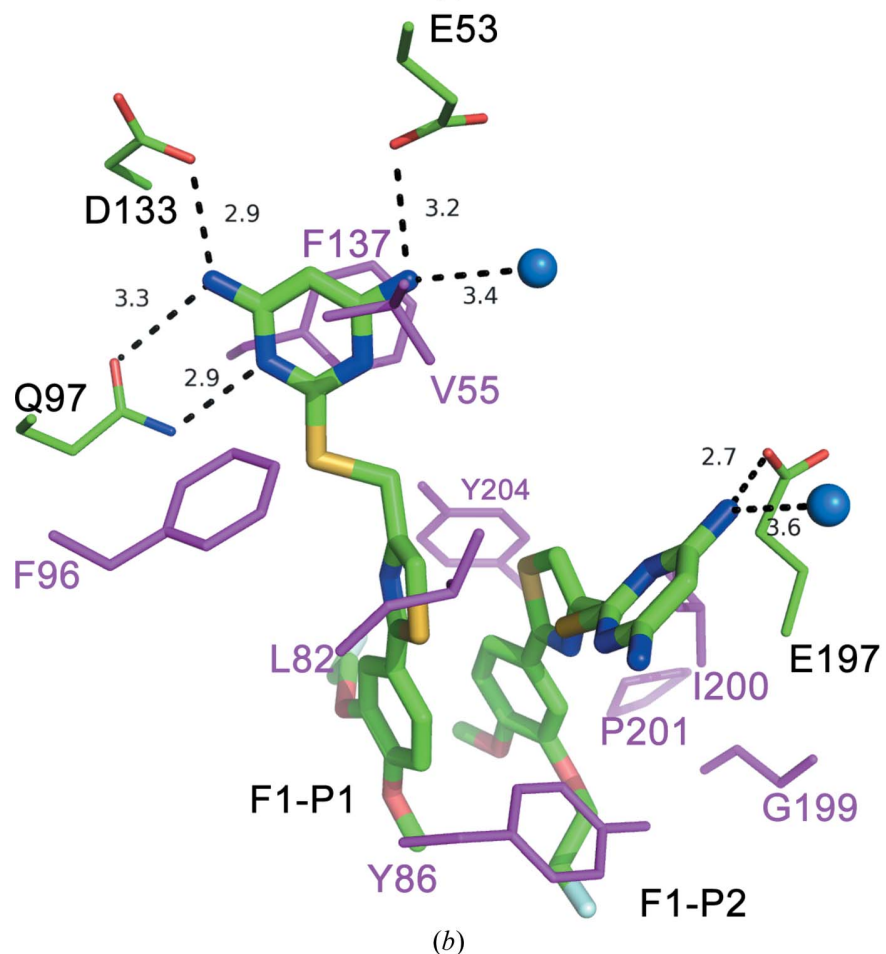

Figure 2

The dCK-F1-UDP complex structure reveals that two molecules of F1 bind to dCK in a parallel manner. (a) The dCK-F1-UDP complex crystallized as a dimer. Protomers $A$ and $B$ of dCK are represented as a cartoon diagram in light and dark green colors, respectively. F1 molecules are depicted in red. This inhibitor binds to the nucleoside-binding site of $\mathrm{dCK}$ in a dimeric fashion and not to the nucleotide-binding site, which is occupied by UDP (magenta). (b) Two F1 molecules (thick green sticks) bind to dCK; the molecule binding deeper into the binding cavity is referred to as F1-P1, while the second molecule is referred to as F1-P2. Residues from dCK implicated in the F1 hydrogen-bond network are represented as thin green sticks. This involves residues Glu53, Gln97 and Asp133 that contact the pyrimidine ring of F1-P1, while F1-P2 makes only one polar contact with dCK via residue Glu197. Amino acids depicted in magenta are hydrophobic residues also involved in F1 binding to dCK. Dashed lines represent hydrogen bonds and distances are in $\AA$. Two water molecules also interact with F1 and are represented as blue spheres. 


\section{Results and discussion}

\subsection{F-series compounds 1-4 are a new class of high-affinity} selective human dCK inhibitors

Here, we report the steady-state kinetic and structural evaluation of a family of dCK inhibitors. The founding member, F1, was synthesized after identifying two hits from an HTS. The details and synthesis of this compound and others are reported in Murphy et al. (2013). F-series inhibitors are composed of a pyrimidine ring carrying two exocyclic amino groups (Fig. 1a). This pyrimidine ring is linked to a thiazole ring via an S atom followed by a methylene group. Finally, the thiazole ring extends to a phenyl ring carrying a fluoroethoxy group at the meta position and a methoxy moiety at the para position. The rationale behind the $\mathrm{F}$ atom is to enable the tracking of these molecules through positron emission tomography (PET) imaging when the inhibitors are synthesized with the ${ }^{18} \mathrm{~F}$ positron-emitting radioisotope. The sole difference between the four F-series family members presented here is the nature of the substituent at the 5-position of the thiazole ring. Apparent $\mathrm{IC}_{50}\left(\mathrm{IC}_{50}^{\mathrm{app}}\right)$ values of the inhibitors were determined using steady-state kinetics. We measured an $\mathrm{IC}_{50}^{\mathrm{app}}$ of $745 \mathrm{n} M$ for compound $\mathrm{F} 1$ (Figs. $1 b$ and $1 c$ ).

\subsection{Crystal structure of dCK in complex with F1 and UDP}

We solved the structure of dCK in complex with F1 and UDP at a resolution of $2.0 \AA$ using molecular replacement. Data-collection and refinement statistics are presented in Table 1 . The crystals were twinned and contained two protomers of dCK in the asymmetric unit (Fig. 2a). Notably, each dCK protomer had two molecules of F1 bound (depicted as red spheres in Fig. 2a). The F1 molecules are bound in a parallel fashion. Interestingly, in work performed by Lexicon Pharmaceuticals, a deposited dCK-inhibitor structure also contained two molecules in the active site (Jessop et al., 2009). However, in contrast to our observations for F1, the Lexicon inhibitor bound in an antiparallel orientation.

The dCK-F1 complex structure revealed that the inhibitor occupies the nucleoside-binding site. In fact, the nucleotide-binding site is occupied by UDP (shown in magenta in Fig. 2a). Because two molecules of F1 bind to $\mathrm{dCK}$, we refer to the molecule binding deeper in the active site as F1-P1 and to the second molecule as F1-P2.

The binding of F1-P1 to dCK mainly involves polar interactions via the pyrimidine moiety (Fig. $2 b$ ). The catalytic carboxylic acid of the enzyme, Glu53, makes a $3.2 \AA$ interaction with one of the two exocyclic amine groups, while Gln97 makes a bidentate hydrogenbond interaction: $2.9 \AA$ to the ring $\mathrm{N}$ atom and $3.3 \AA$ to the second exocyclic amine group. This amine group also makes a $2.9 \AA$ hydrogen bond to Asp133. The most noticeable hydrophobic interaction between F1-P1 and dCK involves a $\pi-\pi$ electron stacking interaction of Phe137 and the pyrimidine ring of the inhibitor. Additional hydrophobic residues also contributing to $\mathrm{F} 1-\mathrm{P} 1$ binding are represented in magenta in Fig. 2(b).

F1-P2, on the other hand, makes noticeably fewer direct contacts with dCK. The lone polar contact seems to be a $2.7 \AA$ interaction with Glu197. Even though the fluoroethoxy chain of F1-P2 is close to several residues (Gly199, Ile200 and Pro201), this second inhibitor molecule seems to mostly bind through a stacking interaction with the thiazole and phenyl rings of F1-P1.

Superimposition of the dCK-F1-UDP complex and the dCK structure in complex with UDP and 5-bromo-deoxycytidine (5Br-dC; PDB entry 3qen) reveals that the pyrimidine rings of $\mathrm{F} 1-\mathrm{P} 1$ and $5 \mathrm{Br}-\mathrm{dC}$ bind in exactly the same position. Moreover, the pyrimidine ring of F1-P1 displays the same hydrogen-bond network with $\mathrm{dCK}$ as that made by the base of the nucleoside (Fig. 3).

\subsection{Compound F1 stabilizes the open state}

Our previous work has reported that $\mathrm{dCK}$ can adopt open and closed states (Godsey et al., 2006; Hazra et al., 2011; Sabini et al., 2007; Sabini, Hazra, Konrad et al., 2008; Sabini, Hazra, Ort et al., 2008), where the closed state is the catalytically competent conformation. The open state allows nucleoside binding/product release but is catalytically incompetent. The nature of the nucleotide (ATP versus UTP) and the phosphorylation state of Ser74 influence the equilibrium between the open and closed states of the enzyme. Whereas ATP/ADP favors the closed dCK conformation, UTP/UDP and phosphorylated Ser74 (as manifested by the S74E mutation) favor the open state (Hazra et al., 2011). We were only able to obtain dCK-inhibitor complex crystals with UDP. We were also more

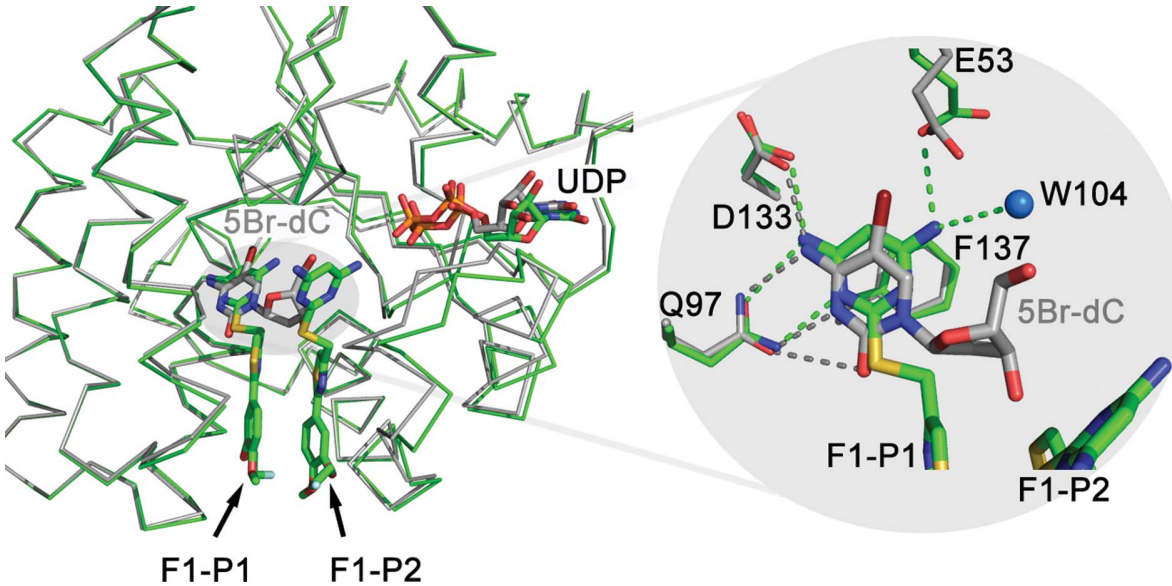

Figure 3

F1 occupies the nucleoside-binding site of dCK. The left panel shows an overlay of dCK-F1-UDP (green ribbon) with dCK-5Br-dC-UDP (gray ribbon; PDB entry 3qen; r.m.s.d. of $0.36 \AA$ over 196 $\mathrm{C}^{\alpha}$ atoms). The enlargement in the right panel demonstrates the nearly perfect overlay of the pyrimidine rings of $\mathrm{F} 1$ and $5 \mathrm{Br}-\mathrm{dC}$ and a conserved hydrogen-bond network (represented as dashed lines). 
successful in reproducibly obtaining good diffracting crystals using the S74E dCK mutant. Hence, we were not surprised to discover that $\mathrm{F} 1$ binds to $\mathrm{dCK}$ in the open state and stabilizes the enzyme in this inactive conformation (Fig. 4). Despite the fact that the S74E mutation promotes the open state, our structure in this conformation is not an artifact solely owing to this modification. This conclusion is supported by the fact that we were able to crystallize and solve the structure of the complex with a non-Ser74 mutant form of dCK and it too adopted the dCK open state (data not shown). Moreover, the dCK-inhibitor structure reported by Lexicon Pharmaceuticals (Jessop et al., 2009; PDB entry 3ipy) also presents dCK in the open conformation, a fact that was not mentioned in their report. Similar to our observation with compound F1, the

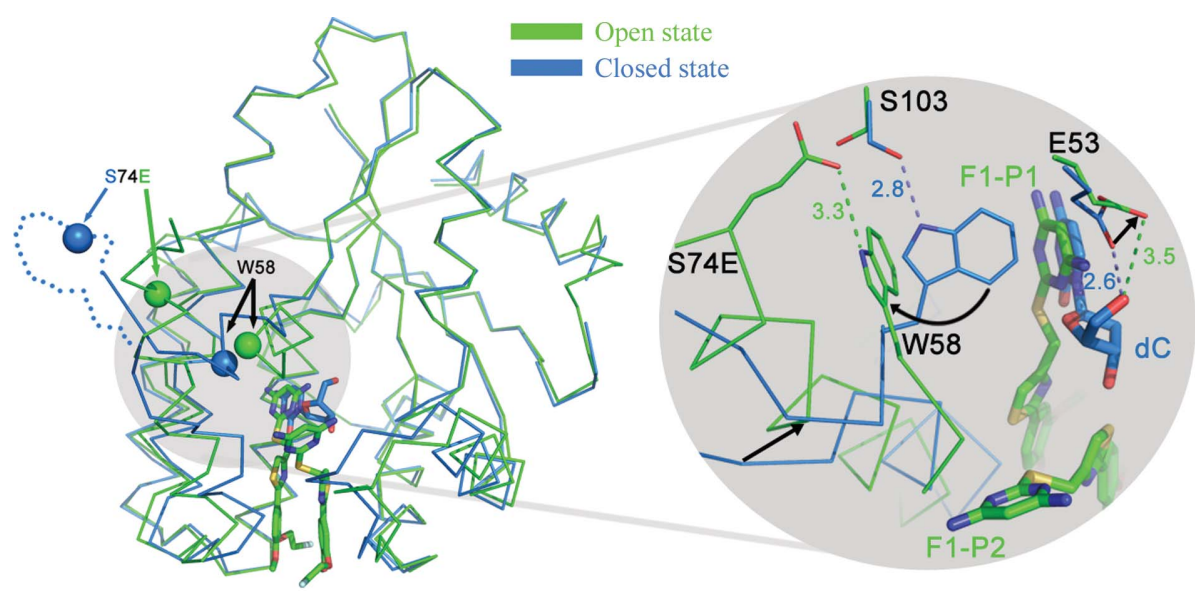

(a)
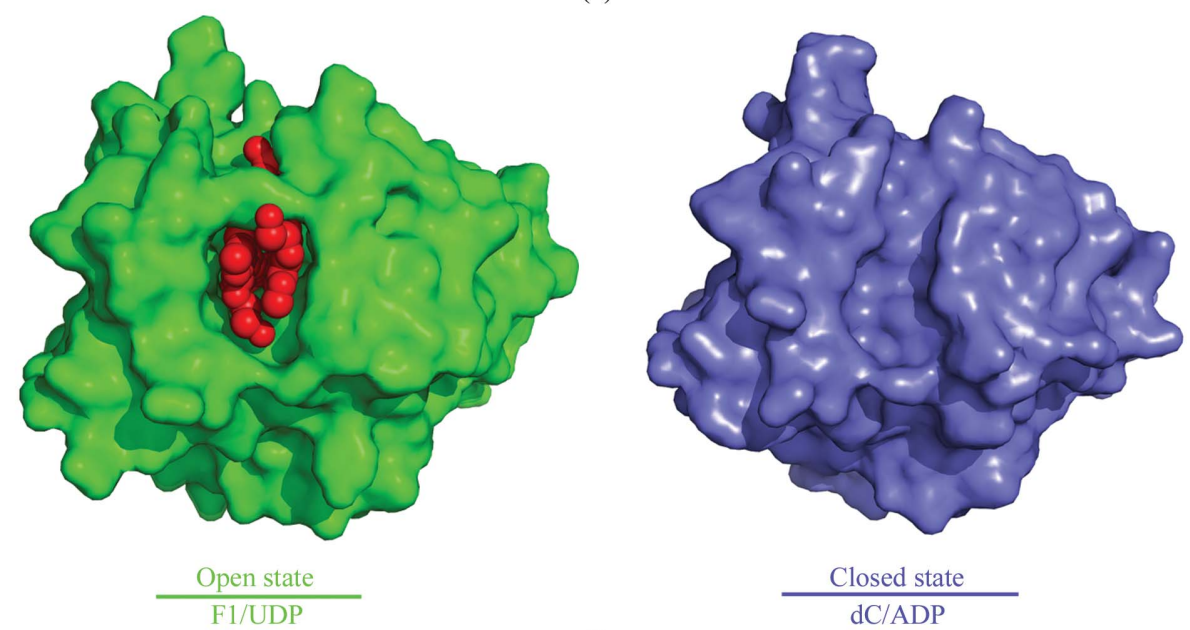

(b)

Figure 4

F1 binds and stabilizes dCK in the open state. (a) dCK-F1-UDP (green ribbon, open state) is overlaid on dCK in complex with deoxycytidine (dC) and ADP (r.m.s.d. of $0.50 \AA$ over $144 \mathrm{C}^{\alpha}$ atoms), which represents the closed state (blue ribbon; PDB entry 2no1). The left panel shows how the loop carrying Ser74 is affected upon phosphorylation. Glu74 (the phosphomimetic residue of the phosphorylated Ser74) is now in contact distance with Trp58. The enlargement on the right displays in detail how the Trp58 side chain reorients and makes a new interaction with Glu74. Black arrows indicate the major transitions required between the closed and open states. Distances are in A. (b) Surface representation of dCK in complex with F1 and UDP (green color with F1 depicted as red spheres; representing the open state) and $\mathrm{dCK}$ in complex with $\mathrm{dC}$ and ADP (blue color; representing the closed state) in the same orientation. In the closed state, the nucleoside is occluded and cannot dissociate from the enzyme. The larger F1 would not fit in the closed enzyme state and must bind to the open state that allows it to protrude into the solvent.
Lexicon compound also bound as two molecules per dCK protomer. Together, this demonstrates that the dCK open in to inhibition by small molecules. ADP (PDB entry 2no1; closed enzy 2007) depicts the differences between the two conformations (Fig. 4). Although the two structures show a good overall fit (r.m.s.d. of $0.51 \AA$ over $144 \mathrm{C}^{\alpha}$ atoms), we can easily differentiate the open and closed states of the enzyme. When dCK transitions to the open conformation, several events occur around the nucleoside-binding site. In terms of catalytic competency, the residue responsible for activating the 5 '-hydroxyl group of the nucleoside for nucleophilic attack on ATP/UTP (2.6 ̊ distance; enlargement in Fig. 4a), Glu53, is now too distant to interact directly with the $5^{\prime}$-hydroxyl group of the nucleoside (3.5 ̊ distance; enlargement in Fig. $4 a$ ). In terms of active-site rearrangement, major changes occur in the vicinity of Trp58. While making an interaction with Ser103 in the closed state, the position of the Trp58 side chain is dramatically changed in the open state, directly interacting with Glu74, the phosphomimetic residue of phosphorylated Ser74. Fig. 4(a) demonstrates the conformational differences between the open and closed dCK states, with a focus on Trp58 and Glu74. The interaction between Glu74 and Trp58 in the open state, built upon the rotation of the Trp58 indole side chain, results in a larger cavity within the dCK active site. The cavity is exploited by F1 when binding to the enzyme (Fig. $4 b$; dCK surface colored in green and F1 depicted in red). In contrast, solvent access to this cavity is obstructed in the closed state of the enzyme (Fig. $4 b$; dCK surface colored in blue). This shows that steric considerations require the binding of F1 to the open dCK state.

The presence of two inhibitor molecules per dCK active site raises several questions. (i) Does this type of inhibitor require the binding of two molecules to achieve an interaction with dCK? (ii) Is the binding sequential or do the inhibitors bind as a dimeric unit? (iii) If the binding is sequential, is the affinity of one molecule stronger than the other? (iv) Lastly, would there be an advantage in generating an inhibitor that binds as a single molecule to dCK? The dCK-F1 complex structure suggests that the 
binding to position 1 is stronger than the binding to position 2 . However, it is not clear whether the inhibitor-inhibitor stacking interaction is a prerequisite for binding to $\mathrm{dCK}$, nor does it clarify the other questions. The additional structures and kinetic analysis presented below provide strong clues to address these questions.

\subsection{Enhancing compound F1 activity against dCK}

The F1 molecule was originally designed with an $\mathrm{H}$ atom at the 5-position of the thiazole ring. While analyzing the dCKF1 complex structure, it became apparent that the F1-P1 thiazole ring is placed such that its 5-position is in the vicinity of an unfilled hydrophobic space (Fig. 5). This suggested that inhibitor binding could be improved by adding a hydrophobic substituent that fills this space. Thus, we progressively extended the substituent of the 5-position of the thiazole ring from the original $\mathrm{H}$ atom (F1) to a methyl, ethyl or propyl moiety (compounds F2, F3 and F4, respectively).

Remarkably, the F2 derivative, which contained a methyl group in place of the $\mathrm{H}$ atom on the thiazole ring, exhibited an $\mathrm{IC}_{50}^{\mathrm{app}}$ of $26 \mathrm{n} M$, a 30-fold decrease relative to $\mathrm{F} 1$. The presence of a longer substituent such as an ethyl (F3) or propyl (F4) moiety resulted in molecules with very similar $\mathrm{IC}_{50}^{\text {app }}$ values (Fig. 1c). It is important to note that the fit of the steady-state kinetic data for the calculation of $\mathrm{IC}_{50}^{\mathrm{app}}$ values assumes a simple hyperbolic inhibition of the enzyme activity. This assumption is valid when the inhibitor affinities have inhibition constant $\left(K_{\mathrm{i}}\right)$ values higher than the enzyme concentration used in the experiment. Since we used a concentration of $50 \mathrm{n} M \mathrm{dCK}$ in our assays (see $\$ 2$ ), this assumption holds true

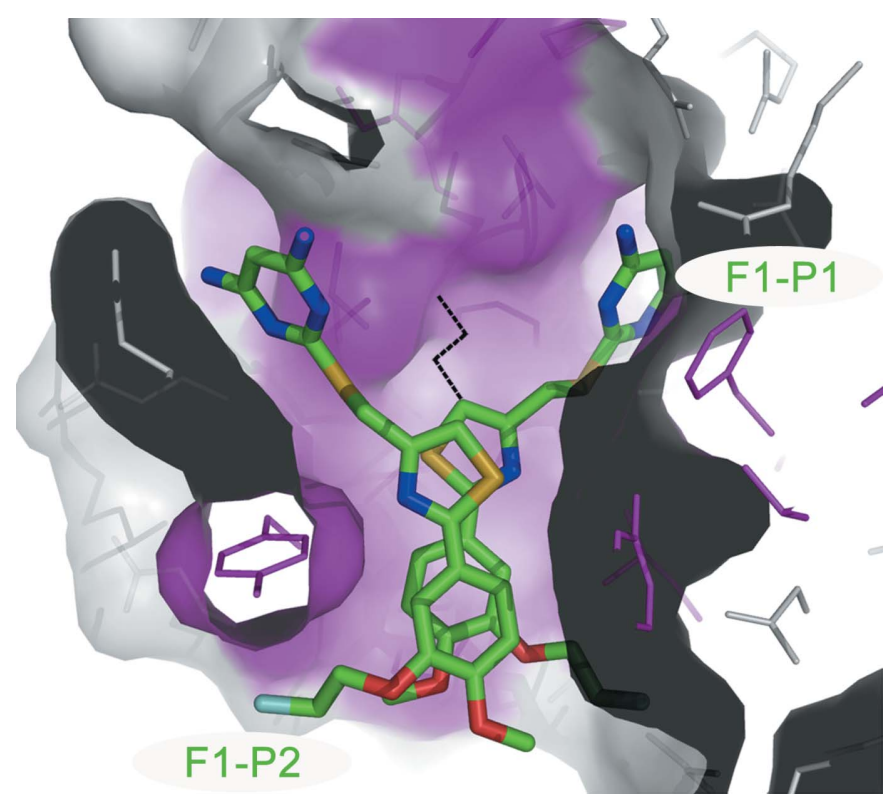

Figure 5

An unfilled hydrophobic space in the proximity of the thiazole ring 5-position. The surface of dCK is shown with hydrophobic residues colored in magenta. The 5-position of the thiazole group of F1-P1 is facing towards a hydrophobic space. This pocket is deep enough to accommodate an alkyl chain at the 5-position of the thiazole ring (represented as a black dashed line). for F1 but not for the other three inhibitors. The latter bind sufficiently tightly that nearly all added inhibitor binds to the enzyme active site until saturation of the enzyme is approached. Hence, the high-affinity inhibitors are binding stoichiometrically to the enzyme for the first half of the titration and only after $50 \%$ of the enzyme is inactivated can one discern differences in inhibitor affinity. This analysis explains why the $\mathrm{IC}_{50}^{\mathrm{app}}$ values for the high-affinity inhibitors are close to $25 \mathrm{n} M$ and why the fit of the data $\left(R^{2}\right)$ becomes progressively worse: as the binding becomes tighter, the deviation from the hyperbolic equation increases.

The breakdown of the empirical approach prompted us to fit the data to a more explicit inhibition model. The model that we used assumes that (i) the binding of one inhibitor molecule to each enzyme active site results in inactivation and that (ii) the inhibitor binds to the active site of each protomer independently so that the binding of the inhibitor to one protomer active site has no effect on the binding to the other active site. This is the simplest type of enzyme-inhibition model; the quadratic equation used (see \$2) reduces to the simple hyperbolic equation when the apparent inhibition constant

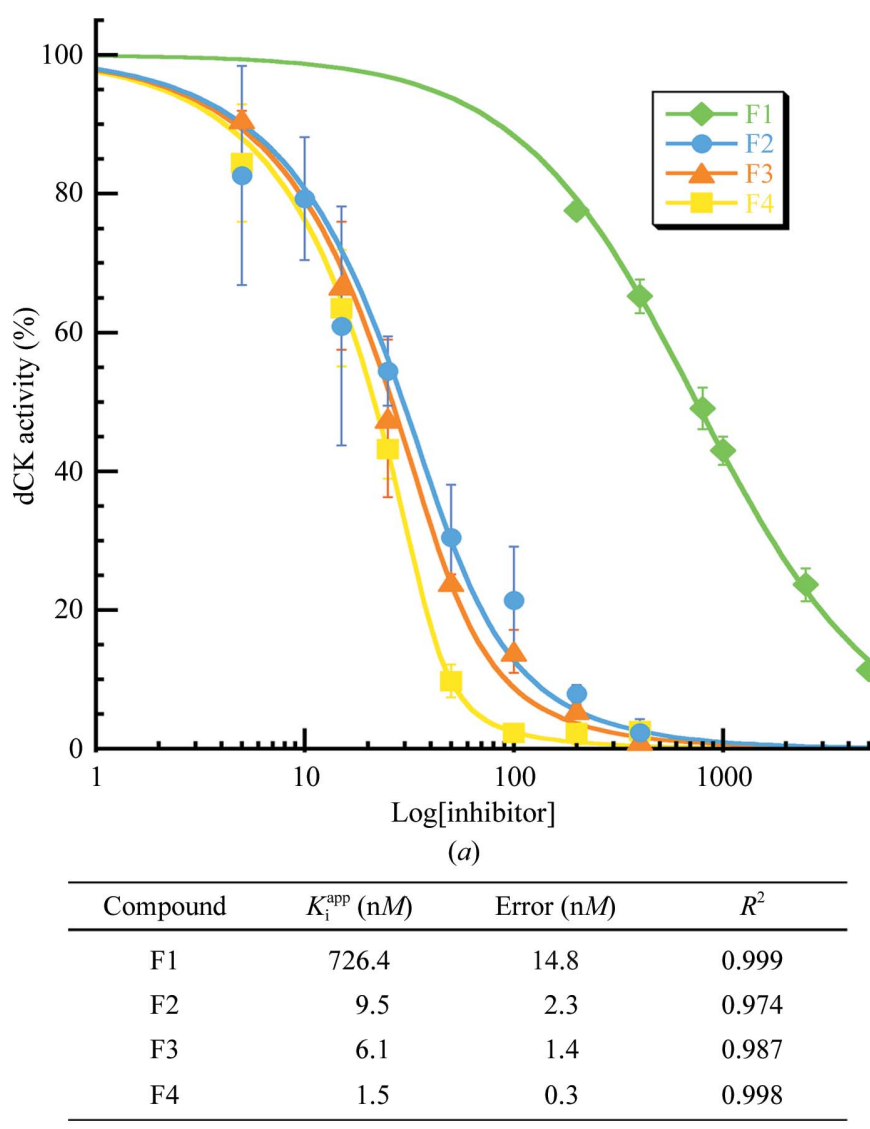

(b)

Figure 6

Determination of $K_{\mathrm{i}}^{\text {app }}$ values better reflects the potency of the inhibitor compared with $\mathrm{IC}_{50}^{\mathrm{app}}$ values. (a) Steady-state data and curve fit with the explicit binding model for the four inhibitors. (b) $K_{\mathrm{i}}^{\text {app }}$, error and coefficient of determination $\left(R^{2}\right)$. The fit to the explicit model (providing $K_{\mathrm{i}}^{\text {app }}$ values) is superior to the simplistic fit that determines IC $_{50}^{\text {app }}$ values, and notably reveals that the inhibition is improved with increased alkylchain length at the 5-position of the thiazole ring. 
$\left(K_{\mathrm{i}}^{\text {app }}\right)$ is much higher than the enzyme concentration used when titrating the enzyme with the inhibitor.

Fitting of the steady-state data to the explicit inhibition model resulted in improved fits, and importantly revealed differences in affinity between the inhibitors. The binding does indeed become increasingly tighter (lower $K_{\mathrm{i}}^{\text {app }}$ ) as the length of the alkyl chain is increased (Fig. 6). To better understand the molecular reasons for the increased inhibition, we solved crystal structures of dCK in complex with these F-compounds (Fig. 7).

\subsection{Crystal structure of dCK in complex with F2 and UDP}

The dCK-F2-UDP complex also crystallized in space group $P 4_{1}$ and contained two protomers of $\mathrm{dCK}$ and four F2 molecules in the asymmetric unit. These structures of the dCK-F1

\begin{tabular}{clcccc}
\hline & & \multicolumn{3}{c}{ Protomer $A$} & Protomer $B$ \\
\cline { 3 - 6 } Compound & \multicolumn{1}{c}{$R=$} & $\mathrm{P} 1$ & $\mathrm{P} 2$ & $\mathrm{P} 1$ & $\mathrm{P} 2$ \\
\hline $\mathrm{F} 1$ & $-\mathrm{H}$ & 1 & 1 & 1 & 1 \\
F2 & $-\mathrm{CH}_{3}$ & 1 & 1 & 1 & 1 \\
F3 & $-\mathrm{CH}_{2}-\mathrm{CH}_{3}$ & 1 & 0.5 & 1 & 0 \\
F4 & $-\mathrm{CH}_{2}-\mathrm{CH}_{2}-\mathrm{CH}_{3}$ & 1 & 0 & 1 & 0 \\
\hline
\end{tabular}
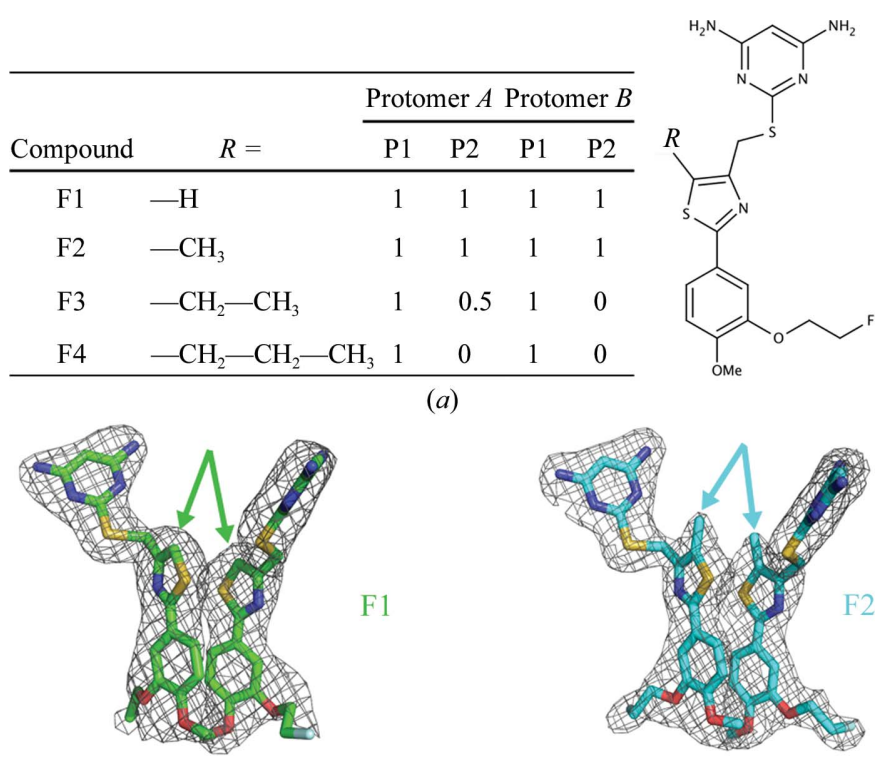

(a)
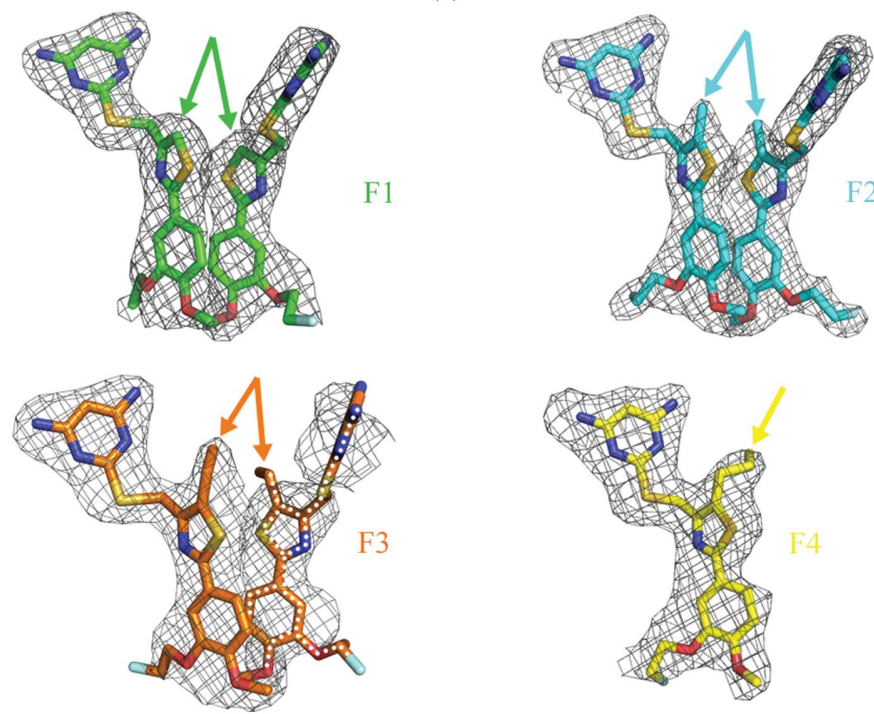

F1

\section{Figure 7}

The nature of the inhibitor determines the occupancy at the active site. (a) The occupancy of F-series molecules at the dCK active site is affected by the substituent at the thiazole ring. We progressively extended an alkyl chain (from a methyl group to a propyl group) at the 5-position of the thiazole ring. The number of inhibitor molecules observed in each $\mathrm{dCK}$ protomer is also shown. F1 and F2 both bind with two molecules per protomer of dCK (protomers $A$ and $B$ ). In contrast, $\mathrm{F} 3$ has 1.5 molecules bound to dCK protomer $A$, which means that $\mathrm{P} 1$ is fully present but the P2 molecule is only observed at an estimated 0.5 occupancy. F3-P2 was absent in dCK protomer $B$. F4 showed only one molecule bound to each dCK protomer. (b) Electron-density OMIT maps $\left[m F_{\mathrm{o}}-D F_{\mathrm{c}}\right.$ as

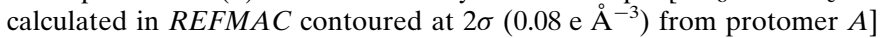
at the position of the inhibitors. The white dotted representation of F3 depicts its partial occupancy. F4 shows only one molecule bound into the active site. and the dCK-F2 complexes display high similarity (r.m.s.d. of $0.33 \AA$ over $208 \mathrm{C}^{\alpha}$ atoms). Owing to the methyl group on the thiazole ring, the $\mathrm{F} 2-\mathrm{P} 1$ position changes by $\sim 0.8 \AA$ relative to the F1-P1 position, whereas F1-P2 and F2-P2 display a better overlay (Fig. 8a). The parallel binding mode of the two inhibitor molecules in positions 1 and 2 is maintained, as seen for F1. This places the thiazole methyl group of one F2 molecule in proximity to the methyl group of the other F2 molecule. As predicted by the structure of the dCK-F1 complex (Fig. 5), the methyl group present in F2-P1 points into the hydrophobic space. This added interaction between F2-P1 and dCK, plus the stacking of the methyl groups of the two bound inhibitor molecules, may explain the increased inhibition of dCK by F2 relative to $\mathrm{F} 1$.

\subsection{Crystal structure of $\mathrm{dCK}$ in complex with F3 and UDP}

Interestingly, despite F2 and F3 crystallizing in the same space group, the structure reveals a different occupancy of the binding sites for the inhibitors while maintaining an identical enzyme conformation. An overlay of the dCK-F1 and dCKF3 complex structures (r.m.s.d. of $0.34 \AA$ over $212 \mathrm{C}^{\alpha}$ atoms; the r.m.s.d. for the overlay of dCK-F2 and dCK-F3 was $0.31 \AA$ over $205 \mathrm{C}^{\alpha}$ atoms) is presented in Fig. 8. In the latter structure, $\mathrm{F} 3$ is clearly present at binding site 1 (in both protomers) as shown by strong electron density (Fig. 7). In contrast, F3 is absent from binding site 2 in protomer $B$ and is only present at low occupancy (estimated to be $\sim 0.5$ ) in protomer $A$ (Figs. 7 and 8). In deciding the presence or absence of F3 at position 2 we are helped by the position adopted by Tyr86. When position 2 is occupied by the inhibitor, as seen for F1 and F2, the side chain of Tyr86 points away from the inhibitor. However, when position 2 is not occupied or is only partially occupied by the inhibitor, Tyr86 rotates $\sim 90^{\circ}$ to occupy a space that would be taken by the pyrimidine ring of the inhibitor. In the dCKF3 complex, the electron density revealed two conformations for Tyr86 in protomer $A$, supporting the observed low F3-P2 occupancy (Fig. $8 b$ ). In protomer $B$, Tyr86 adopted the conformation not consistent with F3-P2 binding, confirming the absence of F3-P2. Hence, the electron density for Tyr86 allows us to confidently assign the presence/absence or partial occupancy of the inhibitor at position 2 .

The binding of a single F3 molecule to dCK answers one of the previously raised questions with an affirmative: these molecules can bind in a single fashion to $\mathrm{dCK}$, which also suggests that the stacking interaction between two inhibitor molecules is not critical. We rationalize the lack/partial binding of $\mathrm{F} 3$ at position 2 by the presence of a second $\mathrm{C}$ atom on the thiazole-ring alkyl chain. The binding of F3-P1 positions the ethyl group pointing towards the $\mathrm{P} 2$ binding site. In this scenario, a second inhibitor molecule is hindered from the $\mathrm{P} 2$ binding site, explaining the partial occupancy in protomer $A$ and its absence in protomer $B$. The observation that enzyme inhibition is correlated with the stoichiometric binding of one F2 per protomer active site implies that the second inhibitor molecule binds more weakly than, and subsequent to, binding of the first molecule. Therefore, the measured $K_{\mathrm{i}}^{\text {app }}$ for F2 


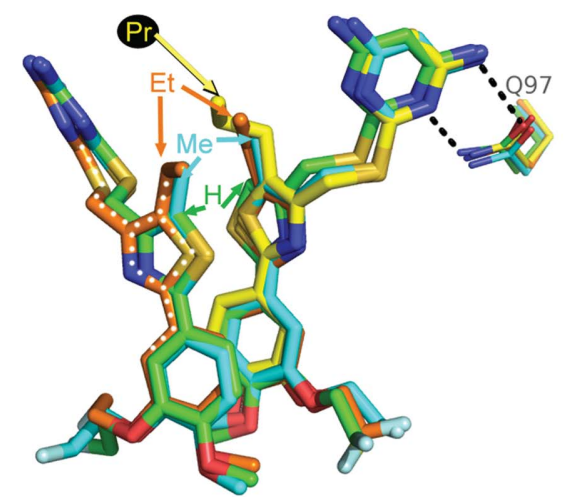

(a)
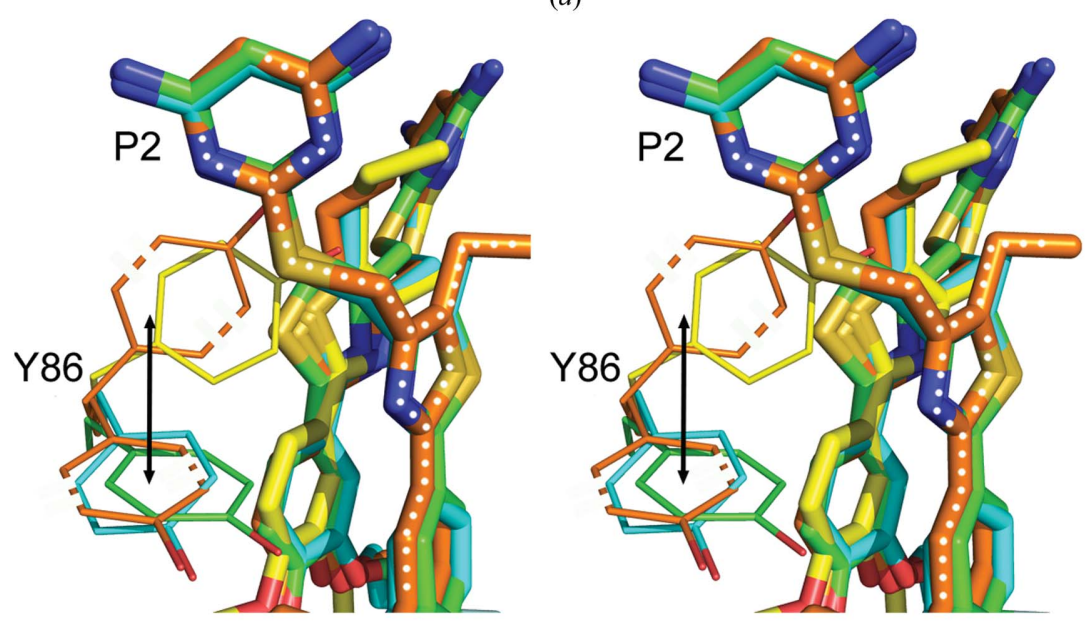

(b)

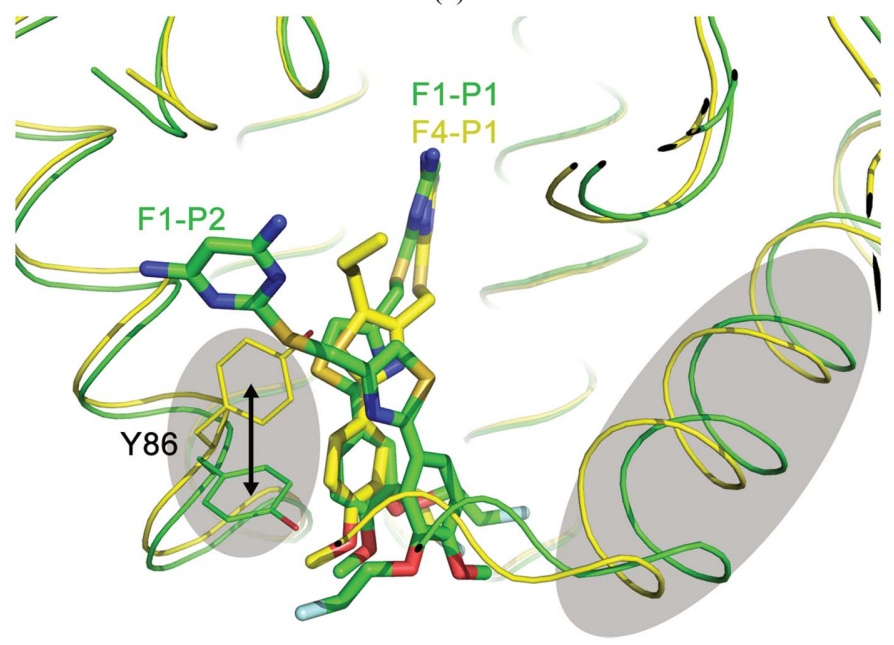

(c)

Figure 8

F-series compounds show different occupancies. (a) Stereoview of F1 (green), F2 (cyan), F3 (orange) and F4 (yellow) compounds after overlaying the corresponding dCK-F-series structures. The colored arrows indicate the component (hydrogen, methyl, ethyl and propyl) at the 5-position of the thiazole ring. The dotted line indicates the F3-P2 molecule observed with partial occupancy. Gln97 interacting with P1 molecules is also represented. (b) Enlargement of the P2 molecules and stereoview representation of the overlaid inhibitors. The position of Tyr86 depends on the number of inhibitor molecules in the active site. Tyr86 faces downwards to accommodate two molecules of F1 and F2. In the dCK-F3 structure, because of the partial occupancy of the P2 molecule, Tyr86 displays two conformations (represented as dashed bonds) that are related by the black arrow. In contrast, Ty 86 fully rotates $\sim 90^{\circ}$ and faces upwards to occupy the space of the missing F4-P2 molecule. (c) The dCK nucleoside-binding site is flexible. The dCK-F1 (green ribbon) and dCK-F4 (yellow ribbon) structures are overlaid. Gray areas highlight the main conformational changes upon binding of one or two inhibitor molecules in the active site of dCK. (i) The black arrow relates the two positions Tyr86 can adopt. (ii) The other main change occurs in the loop spanning residues 200210. Indeed, this loop moves back (green color) when two inhibitor molecules are bound to dCK. reflects the affinity of just the first inhibitor molecule that binds. This affinity may be enhanced by the binding of the second inhibitor molecule. It follows that the lower $K_{\mathrm{i}}^{\text {app }}$ of F3 compared with $\mathrm{F} 2$ results from the greater hydrophobic interaction of the ethyl group than the methyl group.

\subsection{Crystal structure of dCK in complex with F4 and UDP}

Finally, we solved the dCK-F4-UDP complex structure. Like the previous complexes, it crystallized in the same space group with twinning and contained two dCK protomers in the asymmetric unit. Notably, only a single F4 molecule is observed per dCK protomer (Figs. 7 and 8). As discussed earlier, the conformation of Tyr86 unambiguously confirmed the absence of an F4 molecule at the P2 binding site (Figs. $8 b$ and $8 c$ ). This structure demonstrates that the presence of a longer alkyl chain at the 5-position of the thiazole ring increases the binding at $\mathrm{P} 1$ but prevents binding at $\mathrm{P} 2$. The enzyme responds to the lack of a second inhibitor molecule at $\mathrm{P} 2$ by adopting a more compact open state (Fig. 8c). F4 is the most potent inhibitor of this F-series, showing a $K_{\mathrm{i}}^{\mathrm{app}}$ of $1.5 \mathrm{n} M$. Thus, it is clear that only one molecule per protomer is sufficient to fully inhibit dCK activity.

\section{Conclusion}

We elucidated the crystal structures of human dCK in complex with smallmolecule inhibitors. The compounds, referred to as the F-series, occupy the nucleoside-binding site of dCK and do not preclude nucleotide binding. The pyrimidine moiety of the inhibitor binds at the same position and conserves the same hydrogen-bond network as the pyrimidine ring of deoxycytidine, the nucleoside substrate of dCK. Inhibitor binding is to the open form of $\mathrm{dCK}$, which is a nucleoside competent binding/product-release conformation but catalytically inactive state. In fact, the dCK closed state is not competent for inhibitor binding. Taken together with previous work in which a small 
inhibitor molecule was crystallized in complex with dCK in the open state (Jessop et al., 2009), our results support the conclusion that the open state is more vulnerable to inhibition by small molecules.

We examined four related inhibitors that differ in the nature of the substituent located at the 5-position of the thiazole ring. Compound $\mathrm{F}$, with an $\mathrm{H}$ atom at this position, binds in a partially parallel dimeric fashion to $\mathrm{dCK}$, with the thiazole and phenyl rings of one inhibitor molecule stacking against the corresponding rings from the second inhibitor molecule. The pyrimidine rings of the inhibitors do not stack; each binds at a different site. Notably, the pyrimidine ring binding to position 1 makes significantly more enzyme interactions than the corresponding pyrimidine ring binding to position 2 . A hydrophobic space in the proximity of the thiazole ring of the inhibitor at position 1 suggested that molecules with an alkyl substituent would bind more tightly. Indeed, F2, with a methyl group at the 5-position of the thiazole, has a 75-fold lower $K_{\mathrm{i}}^{\text {app }}$. Extending the alkyl substituent to an ethyl (F3) or propyl (F4) group resulted in even tighter binding. Unexpectedly, the molecules with the longer alkyl chain bind singly.

Based on the four dCK-F-series inhibitor complex crystal structures detailed here, we can make the following conclusions. (i) The molecules bind to dCK in either a dimeric or a monomeric fashion, depending on the substituent at the 5 -position of the thiazole ring. (ii) When binding two molecules, the molecule at position 1 binds first and with a higher affinity than the molecule at position 2. (iii) The stacking interaction built between two inhibitor molecules may enhance inhibitor binding affinity but requires inhibitor concentrations much greater than the $K_{\mathrm{i}}^{\text {app }}$. (iv) The lack of a hydrophobic substituent at the 5-position of the thiazole is highly detrimental to affinity (75-fold reduced affinity of F1 compared with F2) owing to an unfilled hydrophobic space; longer chains result in only modestly increased affinity (sixfold increased affinity for F4 over F2). Finally, (v) for the purpose of improving our best binder, F4, future work should focus on modifying the phenyl ring that carries the fluoroethoxy chain, as well as on modifications that will prevent the possible hydrolysis of the thiomethyl thiazole.

This work was supported by the National Institutes of Health (grant R01 EB013685 to AL), the In Vivo Cellular and Molecular Imaging Centers Award, National Institutes of Health (P50 CA86306 to CGR) and the US National Cancer Institute (grant 5U54 CA119347 to CGR). The authors declare the following competing financial interest(s): CGR is a co-founder of Sofie Biosciences, a molecular diagnostic company. He holds equity in Sofie Biosciences. The University of California also holds equity in Sofie Biosciences. Intellectual property that CGR invented and which was patented by the University of California has been licensed to Sofie Biosciences. The University of California has patented additional intellectual property invented by CGR, JMM, ALA and AL. We thank Nagichettiar Satyamurthy for his contributions. Diffraction data for the dCK-F2-UDP complex were collected on the Southeast Regional Collaborative Access
Team (SER-CAT) 22-BM beamline at the Advanced Photon Source (APS), Argonne National Laboratory. Supporting institutions may be found at http://www.ser-cat.org/ members.html. Diffraction data for the other three complexes were collected at the Life Sciences Collaborative Access Team (LS-CAT) at the APS. Use of the LS-CAT Sector 21 was supported by the Michigan Economic Development Corporation and the Michigan Technology Tri-Corridor (Grant 085P1000817). Use of the APS, an Office of Science User Facility operated for the US Department of Energy (DOE) Office of Science by Argonne National Laboratory, was supported by the US DOE under Contract No. DE-AC0206CH11357.

\section{References}

Agarwal, K. C., Miech, R. P. \& Parks, R. E. (1978). Methods Enzymol. 51, 483-490.

Arnér, E. S. \& Eriksson, S. (1995). Pharmacol. Ther. 67, 155-186.

Austin, W. R., Armijo, A. L., Campbell, D. O., Singh, A. S., Hsieh, T., Nathanson, D., Herschman, H. R., Phelps, M. E., Witte, O. N., Czernin, J. \& Radu, C. G. (2012). J. Exp. Med. 209, 2215-2228.

Chen, V. B., Arendall, W. B., Headd, J. J., Keedy, D. A., Immormino, R. M., Kapral, G. J., Murray, L. W., Richardson, J. S. \& Richardson, D. C. (2010). Acta Cryst. D66, 12-21.

Emsley, P., Lohkamp, B., Scott, W. G. \& Cowtan, K. (2010). Acta Cryst. D66, 486-501.

Engh, R. A. \& Huber, R. (1991). Acta Cryst. A47, 392-400.

Eriksson, S., Arnér, E., Spasokoukotskaja, T., Wang, L., Karlsson, A., Brosjö, O., Gunvén, P., Julusson, G. \& Liliemark, J. (1994). Adv. Enzyme Regul. 34, 13-25.

Evans, D. R. \& Guy, H. I. (2004). J. Biol. Chem. 279, 33035-33038.

Fairman, J. W., Wijerathna, S. R., Ahmad, M. F., Xu, H., Nakano, R., Jha, S., Prendergast, J., Welin, R. M., Flodin, S., Roos, A., Nordlund, P., Li, Z., Walz, T. \& Dealwis, C. G. (2011). Nature Struct. Mol. Biol. 18, 316-322.

Godsey, M. H., Ort, S., Sabini, E., Konrad, M. \& Lavie, A. (2006). Biochemistry, 45, 452-461.

Hazra, S., Szewczak, A., Ort, S., Konrad, M. \& Lavie, A. (2011). Biochemistry, 50, 2870-2880.

Jessop, T. C. et al. (2009). Bioorg. Med. Chem. Lett. 19, 6784-6787.

Joachims, M. L., Marble, P. A., Laurent, A. B., Pastuszko, P., Paliotta, M., Blackburn, M. R. \& Thompson, L. F. (2008). J. Immunol. 181, 8153-8161.

Kabsch, W. (2010). Acta Cryst. D66, 125-132.

Kewn, S., Hoggard, P. G., Sales, S. D., Johnson, M. A. \& Back, D. J. (2000). Br. J. Clin. Pharmacol. 50, 597-604.

Laskowski, R. A., MacArthur, M. W., Moss, D. S. \& Thornton, J. M. (1993). J. Appl. Cryst. 26, 283-291.

Montgomery, J. A., Shortnacy-Fowler, A. T., Clayton, S. D., Riordan, J. M. \& Secrist, J. A. III (1992). J. Med. Chem. 35, 397-401.

Murphy, J. M. et al. (2013). J. Med. Chem. 56, 6696-6708.

Murshudov, G. N., Skubák, P., Lebedev, A. A., Pannu, N. S., Steiner, R. A., Nicholls, R. A., Winn, M. D., Long, F. \& Vagin, A. A. (2011). Acta Cryst. D67, 355-367.

Reichard, P. (1988). Annu. Rev. Biochem. 57, 349-374.

Ruiz van Haperen, V. W., Veerman, G., Eriksson, S., Boven, E., Stegmann, A. P., Hermsen, M., Vermorken, J. B., Pinedo, H. M. \& Peters, G. J. (1994). Cancer Res. 54, 4138-4143.

Sabini, E., Hazra, S., Konrad, M. \& Lavie, A. (2007). J. Med. Chem. 50, 3004-3014.

Sabini, E., Hazra, S., Konrad, M. \& Lavie, A. (2008). J. Med. Chem. 51, 4219-4225.

Sabini, E., Hazra, S., Ort, S., Konrad, M. \& Lavie, A. (2008). J. Mol. Biol. 378, 607-621. 


\section{research papers}

Sabini, E., Ort, S., Monnerjahn, C., Konrad, M. \& Lavie, A. (2003). Nature Struct. Biol. 10, 513-519.

Schüttelkopf, A. W. \& van Aalten, D. M. F. (2004). Acta Cryst. D60, 1355-1363.

Tarver, J. E. et al. (2009). Bioorg. Med. Chem. Lett. 19, 6780-6783.

Toy, G., Austin, W. R., Liao, H.-I., Cheng, D., Singh, A., Campbell, D. O., Ishikawa, T. O., Lehmann, L. W., Satyamurthy, N., Phelps, M. E., Herschman, H. R., Czernin, J., Witte, O. N. \& Radu, C. G. (2010). Proc. Natl Acad. Sci. USA, 107, 5551-5556.
Vagin, A. \& Teplyakov, A. (2010). Acta Cryst. D66, 22-25.

Van den Neste, E., Cardoen, S., Offner, F. \& Bontemps, F. (2005). Int. J. Oncol. 27, 1113-1124.

Weng, T., Karmouty-Quintana, H., Garcia-Morales, L. J., Molina, J. G., Pedroza, M., Bunge, R. R., Bruckner, B. A., Loebe, M., Seethamraju, H. \& Blackburn, M. R. (2013). FASEB J. 27, 20132026.

Yu, X.-C., Miranda, M., Liu, Z., Patel, S., Nguyen, N., Carson, K., Liu, Q. \& Swaffield, J. C. (2010). J. Biomol. Screen. 15, 72-79. 